\title{
Metallo- $\beta$-lactamase and genetic diversity of \\ Pseudomonas aeruginosa in intensive care units in \\ Campo Grande, MS, Brazil
}

\begin{abstract}
Infection by Pseudomonas aeruginosa has spread worldwide, with limited options for treatment. The purpose of this study was to investigate metallo- $\beta$-lactamase-producing $P$. aeruginosa strains and compare their genetic profile using samples collected from patients in intensive care units. Forty $P$. aeruginosa strains were isolated from two public hospitals in Campo Grande, Mato Grosso do Sul State, from January $1^{\text {st }}, 2007$ to June $31^{\text {st }}, 2008$. Profiles of antimicrobial susceptibility were determined using the agar diffusion method. Metallo- $\beta$-lactamase was investigated using the double-disk diffusion test and PCR. Molecular typing was performed by pulsed-field gel electrophoresis (PFGE). Respiratory and urinary tracts were the most common isolation sites. Of the 40 samples tested, $72.5 \%$ (29/40) were resistant to ceftazidime and $92.5 \%(37 / 40)$ to imipenem, whereas $65 \%(26 / 40)$ were resistant to both antimicrobials. Fifteen pan-resistant samples were found. Five percent $(2 / 40)$ of samples were positive for metallo- $\beta$-lactamase on the phenotype test. No metallo- $\beta$-lactamase subtype was detected by PCR. Macrorestriction analysis revealed 14 distinct genetic patterns. Based on the superior accuracy of PCR, it can be inferred that $P$. aeruginosa isolates from the investigated hospitals have alternative mechanisms of carbapenem resistance. The results also suggest clonal spread of $P$. aeruginosa between the studied hospitals.
\end{abstract}

Keywords: Pseudomonas aeruginosa; drug resistance, multiple; beta-lactamases; electrophoresis; gel; pulsed-field.

[Braz J Infect Dis 2011;15(3):195-199]@Elsevier Editora Ltda.

\section{INTRODUCTION}

Pseudomonas aeruginosa, a non-fermenting Gram-negative rod of great clinical and epidemiological relevance in hospital-acquired infections, is more frequently found in intensive care units (ICUs) ${ }^{1}$ and is associated with high morbimortality. $^{2}$

The selective pressure exerted by the indiscriminate use of antimicrobial agents in the last decades might have contributed to the emergence of microorganisms that are resistant to different classes of antibiotics and is currently a matter of worldwide concern. ${ }^{3}$ Several mechanisms are involved in $P$. aeruginosa resistance to antimicrobial agents, such as chromosomal expression, $\beta$-lactamase production, efflux pumps and decrease in membrane permeability. ${ }^{4}$

The currently observed increased resistance to carbapenem has stimulated studies aiming at a better understanding of the resistance mechanisms of $P$. aeruginosa. $3,5,6$ The emergence and dissemination of metallo- $\beta$-lactamases (MBL) have contributed to the high rate of resistance among the $P$. aeruginosa strains isolated in the last decades. The MBL production knowledge is crucial to implement preventive measures that can curb the expansion of bacteria presenting this potent resistance gene. ${ }^{3}$

The objective of the present study was to investigate metallo- $\beta$-lactamase and genetic similarity among $P$. aeruginosa strains isolated in ICUs of two hospitals in the city of Campo Grande, state of Mato Grosso do Sul, Brazil.

\section{MATERIALS AND METHODS}

\section{Research site and period}

The study was carried out with $P$. aeruginosa strains from patients admitted at adult ICUs, coronary care units (CCUs) and pediatric ICUs (PICUs) of two public hospitals in the state of Mato Grosso do Sul, Brazil, from 01/01/2007 to $06 / 31 / 08$. Hospital A is a 250-bed university hospital and hospital B is a 360-bed tertiary hospital.
Authors

Ana Claudia Souza Rodrigues ${ }^{1}$ Marilene Rodrigues Chang ${ }^{2}$ Gabriela Dorn Nóbrega ${ }^{3}$ Mayara Souza Rodrigues ${ }^{3}$ Nadia Cristina Pereira Carvalho ${ }^{4}$ Beatriz Garcia Gomes ${ }^{5}$ Dênio Lopes de Almeida ${ }^{6}$ Karyne Rangel Carvalho ${ }^{7}$ Marise Dutra Asensi ${ }^{8}$

${ }^{1} \mathrm{MD}$; Teacher, Health and Development Post-graduation Program, Central-West Region, Universidade Federal de Mato Grosso do Sul, MS, Brazil

${ }^{2} \mathrm{PhD}$; Teacher, Department of Pharmacy and Biochemistry, Universidade Federal de Mato Grosso do Sul, MS, Brazil

${ }^{3}$ Pharmacist; Postgraduate student, Department of Pharmacy and Biochemistry, Universidade Federal de Mato Grosso do Sul, MS, Brazil ${ }^{4}$ Pharmacist; Postgraduate student, Hospital Universitário, Universidade Federal de Mato Grosso do Sul, MS, Brazil

${ }^{5}$ Pharmacist; Postgraduate student, Hospital Regional de Mato Grosso do Sul, MS, Brazil

${ }^{6}$ Graduate student; Department of Pharmacy and Biochemistry, Universidade Federal de Mato Grosso do Sul, MS, Brazil

${ }^{7} \mathrm{MD}$; Postgraduate student, Laboratório de Pesquisa em Infecção Hospitalar/IOC/FIOCRUZ, RJ, Brazil ${ }^{8} \mathrm{PhD}$; Researcher, Laboratório de Pesquisa em Infecção Hospitalar/IOC/ FIOCRUZ, RJ, Brazil

Submitted on: $11 / 06 / 2010$ Approved on: 12/21/2010

Correspondence to: Ana Claudia Souza Rodrigues Rua Melanias Barbosa, 380. Bloco 3 ap. 302

Taquarussu, Campo Grande MS/Brasil

Phone: 67- 33319974 / 67-84016036/ (Fax) 33227107 anaclaurod@yahoo.com.br

\section{Financial Support: FUNDECT/} MS - Fundação de Apoio ao Desenvolvimento do Ensino, Ciência e Tecnologia do Estado de Mato Grosso do Sul.

We declare no conflict of interest. 


\section{Inclusion criteria}

$P$. aeruginosa strains resistant to ceftazidime and/or imipenem, isolated from several types of biological material were included in the study. One sample was obtained from each patient and stored in a strain bank at the hospitals. These strains were randomly selected without distinction regarding infection or colonization and were not obtained from surveillance cultures.

\section{Bacterial identification and susceptibility to antibiotic agents}

$P$. aeruginosa isolates were identified using conventional methodology. ${ }^{7}$ The antimicrobial susceptibility was determined by the agar diffusion method, according to the Clinical Laboratory Standard Institute (CLSI) recommendations. ${ }^{8}$ The tested antibiotics were polymyxin - POL $(10 \mu \mathrm{g})$, tazobactam + piperacillin - TZP (100/10 $\mu \mathrm{g})$, imipenem - IPM (10 $\mu \mathrm{g})$, meropenem - MER $(10 \mu \mathrm{g})$, amikacin - AMI $(30 \mu \mathrm{g})$, gentamicin - GEN $(10 \mu \mathrm{g})$, aztreonam - ATM $(30 \mu \mathrm{g})$, cefepime - CPM (30 $\mu \mathrm{g})$, ceftazidime - CAZ $(30 \mu \mathrm{g})$ and ciprofloxacin - CIP $(5 \mu \mathrm{g})$. $P$. aeruginosa strains sensitive to polymyxin B only were considered pan-resistant.

\section{MBL detection}

MBL was detected using a phenotypic disc method as described by Arakawa et al. ${ }^{9}$ with the modifications suggested by Picão et al. ${ }^{10}$ The samples were suspended in saline solution ( 0.5 in the McFarland turbidity scale) and seeded with a sterile swab on an Oxoid Mueller-Hinton Agar dish. The ceftazidime $(30 \mu \mathrm{g})$ and imipenem $(10 \mu \mathrm{g})$ discs were placed at a $2-\mathrm{cm}$ distance from a disc without antibiotic agent, which received $5 \mu \mathrm{L}$ of 2 -mercaptopropionic acid solution diluted 1:8. After incubation at $37^{\circ} \mathrm{C}$ for 16 to 18 hours, samples that presented an increase in halo size around the ceftazidime or imipenem discs were considered positive. A standard strain of $P$. aeruginosa (SPM-1, P1088) was used as positive control, which was kindly provided by ALERTA Laboratory - UNIFESP.

$P$. aeruginosa strains positive for $\mathrm{MBL}$ production at the phenotypic test were submitted to conventional PCR. ${ }^{11}$ The starter oligonucleotides used were: blaSPM-1 F (5'CCT ACA ATC TAA CGG CGA CC3') and R (5'TCG CCG TGT CCA GGT ATA AC3'), blaIMP-1 F (5'CTA CCG CAG CAG AGT CTT TTG3') and R (5'AAC CAG TTT TGC CTT ACC AT3') blaIMP-2 F (5'GTT TTA TGT GTA TGC TTC CTT TGT AGC3') and R (5'CAG CCT GTT CCC ATG TAC G3'), blaVIM-1 F (5'GTT TGG TCG CAT ATC GCA AC3') e R (5’AGA CCG CCC GGT AGA CC3'), blaVIM-2 F (5'ATG AAA GTG CGT GGA GAC3') and R (5'CTA CTC AAC GAC TGA GCG ATT TGT3’). ${ }^{12,13}$

\section{Pulsed field gel electrophoresis}

Genotype determination was carried out by pulsed field gel electrophoresis (PFGE), according to Romao et al. ${ }^{14}$ Chromosomal DNA was prepared using the in situ technique in agarose blocks. A bacterial suspension at the exponential growth phase was added to a $400 \mu \mathrm{L}$ of BSC solution (Tris $1 \mathrm{M}$, pH 8.0, EDTA $0.5 \mathrm{M}$ ). After that, $5 \mu \mathrm{L}$ of proteinase $\mathrm{K}$ (Sigma) and $200 \mu \mathrm{L}$ of $1 \%$ agarose (low melting) were added to the cell suspension. The mixture was distributed in molds. The blocks were transferred to a lyse solution (TRIS $1 \mathrm{M}, \mathrm{pH} 8.0$; EDTA $0.5 \mathrm{M}, \mathrm{pH} 8.0$ and $10 \% \mathrm{~N}$-lauryl sarcosine $+5 \mu \mathrm{L}$ of proteinase $\mathrm{K}$ ) and incubated at $50^{\circ} \mathrm{C}$ for 24 hours. Subsequently, the molds were washed six times with TE buffer at $37^{\circ} \mathrm{C}$ and incubated with enzyme buffer for one hour at $5^{\circ} \mathrm{C}$. After buffer removal, the molds were incubated with restriction enzyme SpeI (Invitrogen) at $37^{\circ} \mathrm{C}$ for 2 hours. The electrophoresis procedure was carried out by PFGE in $1 \%$ agarose gel and run with a buffer containing $0.4 \mathrm{X}$ Tris Borato EDTA in the CHEF DR II system (BioRad, California). The gel run consisted in a crescent pulse time of 5 to $25 \mathrm{~s}$, for 18 hours at $6 \mathrm{~V} / \mathrm{cm}$ at a temperature of $14^{\circ} \mathrm{C}$, with an angle of $120^{\circ}$. The fragments were stained with ethidium bromide and photographed. The band profile was analyzed using the Gel Compar III system (Applied Maths, Belgium). The dendrogram was generated using the UPGMA algorithm.

\section{RESULTS}

A total of 40 strains of $P$. aeruginosa were analyzed, of which 12 were isolated from hospital A and 28 from hospital B. Of the total number of samples, 36 came from the adult intensive care unit (ICU), 3 from the coronary care unit (CCU) and 1 from the pediatric ICU. The samples were isolated from several sites: tracheal aspirate $(17 ; 42.5 \%)$, urine $(13 ; 32.5 \%)$, catheter tip $(4 ; 10 \%)$, blood $(2 ; 5 \%)$ and others $(4 ; 10 \%)$, such as pressure ulcer secretion, sacral ulcer and trochanteric ulcer.

Table 1 shows the percentage of resistance in the presence of the tested antimicrobial agents. Of the total strains, $72.5 \%(29 / 40)$ were resistant to ceftazidime, $92.5 \%(37 / 40)$ to imipenem and 65\% (26/40) had simultaneous resistance to both antimicrobials. Approximately $37.5 \%$ of the strains were considered pan-resistant. All samples were sensitive in vitro to polymyxin $\mathrm{B}$.

Table 1. Antimicrobial resistance of $\mathrm{P}$. aeruginosa in ICUs, Campo Grande/MS

\begin{tabular}{lc}
\hline Antimicrobial agents & Resistance $\%(\mathbf{n}=\mathbf{4 0 )}$ \\
\hline Cefepime & 95 \\
\hline Imipenem & 92.5 \\
\hline Ciprofloxacin & 90 \\
\hline Gentamicin & 85 \\
\hline Aztreonam & 85 \\
\hline Amikacin & 72.5 \\
\hline Ceftazidime & 72.5 \\
\hline Meropenem & 70 \\
\hline Tazobactam + piperacillin & 70 \\
\hline Polymyxin & 00
\end{tabular}

ICUs, intensive care units. 
Table 2 shows 17 different bacterial resistance patterns: six were found in hospital A and 14 in hospital B. Three resistance patterns (numbers 1,8 and 15) were found in both hospitals studied.

The phenotypic test to detect MBL was positive in 5\% $(2 / 40)$ of $P$. aeruginosa isolates; however, none of the genes in- vestigated (bla-IMP, bla-VIM and bla-SPM) was identified by PCR. The molecular analysis generated by the PFGE disclosed 14 distinct genetic patterns (A to N). Table 2 shows some common profiles (A, B, E, F, J, K) at the two hospital institutions. Profile A was the most frequent $(n=9)$, of which one strain was from hospital A and eight from hospital B (Table 3).

Table 2. Phenotypic and molecular profile of P. aeruginosa, Campo Grande/MS

\begin{tabular}{|c|c|c|c|c|}
\hline Profile & Patterns of resistance & Hosp & PFGE & Number of samples \\
\hline \multirow{2}{*}{01} & \multirow{2}{*}{ PPT/AMI/ATM/CPM/CAZ/CIP/GEN/IPM/MER } & $\mathrm{HB}$ & $\mathrm{A} / \mathrm{B} / \mathrm{D} / \mathrm{E}$ & $07 / 1 / 1 / 1$ \\
\hline & & HA & $\mathrm{A} / \mathrm{B} / \mathrm{J}$ & $1 / 2 / 1$ \\
\hline 02 & PPT/AMI/ATM/CAZ/CIP/GEN/IPM/MER & $\mathrm{HB}$ & G & 1 \\
\hline 03 & PPT/CPM/ATM/CAZ/CIP/GEN/IPM & $\mathrm{HB}$ & A & 1 \\
\hline 04 & $\mathrm{CIP} / \mathrm{GEN} / \mathrm{IPM}$ & $\mathrm{HB}$ & $\mathrm{C}$ & 1 \\
\hline 05 & AMI/CPM/CIP/GEN/IPM & $\mathrm{HB}$ & $\mathrm{C} / \mathrm{E}$ & $1 / 1$ \\
\hline 06 & CPM/GEN/CIP/IPM & $\mathrm{HB}$ & $\mathrm{C}$ & 1 \\
\hline 07 & PPT/AMI/ATM/CPM/CAZ/CIP/GEN & $\mathrm{HB}$ & $\mathrm{C}$ & 1 \\
\hline \multirow{2}{*}{08} & \multirow{2}{*}{ AMI/ CPM/ ATM/ CIP/ GEN/ IPM/ MER } & $\mathrm{HB}$ & $\mathrm{B} / \mathrm{C} / \mathrm{K}$ & $1 / 1 / 1$ \\
\hline & & HA & B & 2 \\
\hline 09 & PPT/ ATM/CPM/CAZ/IPM & $\mathrm{HB}$ & $\mathrm{I}$ & 1 \\
\hline 10 & PPT/ ATM/CPM/CAZ/GEN/IPM & $\mathrm{HB}$ & $\mathrm{D}$ & 3 \\
\hline 11 & PPT/ ATM/CPM/CAZ/GEN & $\mathrm{HB}$ & M & 1 \\
\hline 12 & PPT/AMI/CPM/CAZ/CIP/GEN/IPM & $\mathrm{HB}$ & $\mathrm{J}$ & 1 \\
\hline 13 & ATM/CPM/CAZ/CIP/IPM/MER & HA & $\mathrm{N}$ & 1 \\
\hline 14 & PPT/ATM/CPM/CAZ/IPM/MER & $\mathrm{HB}$ & $\mathrm{C}$ & 1 \\
\hline \multirow{2}{*}{15} & \multirow{2}{*}{ PPT/AMI/ATM/CPM/CIP/GEN/IPM/MER } & $\mathrm{HB}$ & $\mathrm{F} / \mathrm{H}$ & $1 / 1$ \\
\hline & & HA & $\mathrm{F}$ & 1 \\
\hline 16 & AMI/CPM/CAZ/CIP/GEN/IPM/MER & HA & $\mathrm{L}$ & 1 \\
\hline 17 & AMI/ATM/CPM/CAZ/CIP/GEN/IPM/MER & HA & $\mathrm{E} / \mathrm{K}$ & $1 / 1$ \\
\hline
\end{tabular}

PFGE, pulsed field gel electrophoresis; AMI, amikacin; ATM, aztreonam; CAZ, ceftazidime; CIP, ciprofloxacin; CPM, cefepime; GEN, gentamicin; IPM, imipenem; MER, meropenem; PPT, piperacillin + tazobactam; POL, polymyxin; Hosp, hospital; HA, hospital A; HB, hospital B.

Table 3. Period and hospital where P. aeruginosa was isolated with PFGE profile A

\begin{tabular}{lccccc}
\hline Register & Hospital & Date & Clinic & Underlying disease & Material \\
\hline 1777 & HB & $09 / 01 / 06$ & Adult ICU & Kidney disease & U \\
\hline 1694 & HB & $21 / 02 / 06$ & Adult ICU & Neurological disease & TA \\
\hline 1695 & HB & $07 / 03 / 06$ & Adult ICU & Neurological disease & TA \\
\hline 1696 & HB & $17 / 04 / 06$ & Adult ICU & Diabetes mellitus & U \\
\hline 1770 & HA & $26 / 6 / 06$ & Adult ICU & Heart disease & U \\
\hline 1701 & HB & $11 / 07 / 06$ & CCU & Respiratory disease & TA \\
\hline 1712 & HB & $27 / 11 / 06$ & Adult ICU & Liver disease & BL \\
\hline 1718 & HB & $23 / 03 / 07$ & Adult ICU & AIDS & CT \\
\hline
\end{tabular}

PFGE, pulsed field gel electrophoresis; ICU, intensive care unit; CCU, coronary care unit; AIDS, acquired immune deficiency syndrome; U, urine; TA, tracheal aspirate; BL, blood ; CT, catheter tip; HA, hospital A; HB, hospital B. 


\section{DISCUSSION}

Patients with preexisting comorbidities, long hospital stay and those submitted to invasive procedures such as catheters and mechanical ventilation make the ICUs propitious places for $P$. aeruginosa dissemination, ${ }^{15,16}$ very often associated with high levels of morbimortality. ${ }^{17}$

$P$. aeruginosa was isolated mainly from materials such as tracheal aspirate and urine, corroborating the literature data reporting that this agent is more often isolated from the lower respiratory and urinary tracts. ${ }^{18-20}$

Resistance to different classes of antibiotics has been attributed to the combination of multiple mechanisms, ${ }^{4}$ which might explain the diversity of resistance patterns shown in Table 2.

The use of the lipopeptide class, such as polymyxin B and colistin, was abandoned for a while due to the high toxicity, but recently, it has once again become an important therapeutic option for multiresistant microorganism infections. ${ }^{21}$ Supporting this concept at the present investigation, all $P$. aeruginosa strains were sensitive to this drug.

The analysis of the susceptibility profile in vitro demonstrated higher levels of resistance in the presence of cefepime, imipenem and ciprofloxacin, drugs used in the treatment of severe Pseudomonas infections. On the other hand, as shown in Table 1, tazobactam in combination with piperacillin, meropenem and ceftazidime were the drugs that demonstrated the lowest resistance, suggesting that these drugs, as well as polymyxin, can be used as therapeutic option for $P$. aeruginosa infection in both institutions. The higher resistance of cefepime when compared to ceftazidime might indicate the presence of OXA-31, an oxacillinase that has cefepime as its preferential substrate. ${ }^{22}$

The dissemination capacity of MBL that hydrolyses expanded-spectrum $\beta$-lactams, including cephalosporins and carbapenems, has been a matter of great concern in the world. Studies in several parts of the world have demonstrated varied rates of MBL production by $P$.aeruginosa, such as $6.2 \%$ in Korea, $635 \%$ in Canada, ${ }^{5}$ and $62 \%$ in Greece. ${ }^{23}$ In Brazil, the SPM subtype has been described as the most prevalent in several regions of the country. National data have reported different rates of MBL production, which vary according to the institution and the studied region. As an example, one could cite rates of $10.9 \%$ in hospitals in Rio Grande do Sul, ${ }^{24} 20 \%$ in Rio de Janeiro - RJ, ${ }^{25}$ and $41.9 \%$ in the state of Goiás. ${ }^{26}$ Figueiredo-Mendes et al. ${ }^{27}$ described higher rates of this enzyme production (77.8\%) among $P$. aeruginosa strains previously resistant to carbapenems in hospitals in São Paulo and Brasília.

Different from what is observed in other Brazilian regions, our series did not show the presence of MBL-producing $P$. aeruginosa. Further studies with larger sample sizes are necessary to detect the emergence of this important resistance gene in hospitals in the state of Mato Grosso do Sul.
The fact that the genes bla-SPM, bla-IMP and bla-VIM were not detected even in samples with a positive phenotypic test for MBL suggests the unreliability of the phenotypic test that was applied.

The finding of identical genetic profiles generated by PFGE in the two hospitals suggests an inter-hospital dissemination of $P$. aeruginosa. The high rate of healthcare professional and patient turnover between the hospitals and hospital wards might have contributed to these results.

The distribution of $P$. aeruginosa with A profile (Table 3) suggests inter-hospital transmission, as it was initially found in the adult ICU and it was subsequently found at the CCU of hospital B. This clone (A profile) was also isolated at different periods, which might indicate that it is endemic in this hospital. It is noteworthy the fact that many strains with this profile were sensitive only to polymyxin $B$, which shows high resistance of strains with this genetic pattern.

\section{CONCLUSION}

The results suggest that the resistance of $P$. aeruginosa to carbapenem agents in the present study is due to resistance mechanisms other than MBL production. The inter-hospital and intra-hospital clonal dissemination is a matter of concern and must be contained through preventive hospital infection control measures. Considering the emergent characteristic of MBL, its surveillance becomes important to control the transmission of this resistance mechanism.

\section{ACKNOWLEDGEMENTS}

To Débora Olartechea de Alencar, Silvana Ulisseia Kamis, Fernando Aguilar, Ligiane Stabullo, Francielle G. Bortoly Carvalho, Elaine C. M. Totino and the staff of the Departments of Microbiology of Hospital Regional de Mato Grosso do Sul and Hospital Universitário de Mato Grosso do Sul. We especially thank Dr. Ana Gales (Laboratory ALERTA/ UNIFESP) and her team for sharing their expertise in the subject and Ana Paula D'Alincourt C. Assef, from the Hospital Infection Research Laboratory of IOC/FIOCRUZ for her support with molecular biology techniques.

Part of the present study was supported by Fundação de Apoio ao Desenvolvimento do Ensino, Ciência e Tecnologia of the State of Mato Grosso do Sul - FUNDECT/MS.

\section{REFERENCES}

1. Agodi A, Barchitta M, Cipresso R, Giaquinta L, Romeo MA, Denaro C. Pseudomonas aeruginosa carriage, colonization, and infection in ICU patients. Intensive Care Med 2007; 33(7):1155-61.

2. Zavascki AP, Barth AL, Goncalves AL et al. The influence of metallo-beta-lactamase production on mortality in nosocomial Pseudomonas aeruginosa infections. J Antimicrob Chemother 2006; 58(2):387-92. 
3. Gales AC, Menezes LC, Silbert S, Sader HS. Dissemination in distinct Brazilian regions of an epidemic carbapenem-resistant Pseudomonas aeruginosa producing SPM metallo-beta-lactamase. J Antimicrob Chemother 2003; 52(4):699-702.

4. Livermore DM. Multiple mechanisms of antimicrobial resistance in Pseudomonas aeruginosa: our worst nightmare? Clin Infect Dis 2002; 34(5):634-40.

5. Pitout JD, Chow BL, Gregson DB, Laupland KB, Elsayed S, Church DL. Molecular epidemiology of metallo-beta-lactamase-producing Pseudomonas aeruginosa in the Calgary Health Region: emergence of VIM-2-producing isolates. J Clin Microbiol 2007; 45(2):294-8.

6. Yong D, Choi YS, Roh KH et al. Increasing prevalence and diversity of metallo-beta-lactamases in Pseudomonas spp., Acinetobacter spp., and Enterobacteriaceae from Korea. Antimicrob Agents Chemother 2006; 50(5):1884-6.

7. Gilardi GL. Practical schema for the identification of nonfermentative gram negative bacteria encountered in medical bacteriology. Am J Med Technol 1972; 38(3):65-72.

8. CLSI. Clinical Laboratory Standard of Institute. Performance standards for antimicrobial susceptibility testing 2007; M100S17.

9. Arakawa Y, Shibata N, Shibayama K et al. Convenient test for screening metallo-beta-lactamase-producing gram-negative bacteria by using thiol compounds. J Clin Microbiol 2000; 38(1):40-3.

10. Picao RC, Andrade SS, Nicoletti AG et al. Metallo-beta-lactamase detection: comparative evaluation of double-disk synergy versus combined disk tests for IMP-, GIM-, SIM-, SPM-, or VIM-producing isolates. J Clin Microbiol 2008; 46(6):2028-37.

11. Mullis K, Faloona F, Scharf S, Saiki R, Horn G, Erlich H. Specific enzymatic amplification of DNA in vitro: the polymerase chain reaction. Cold Spring Harb Symp Quant Biol 1986; $51 \mathrm{Pt}$ 1:263-73.

12. Kimura S, Alba J, Shiroto K et al. Clonal diversity of metallobeta-lactamase-possessing Pseudomonas aeruginosa in geographically diverse regions of Japan. J Clin Microbiol 2005; 43(1):458-61.

13. Jeong $\mathrm{SH}, \mathrm{Bae} \mathrm{IK}$, Park KO et al. Outbreaks of imipenem-resistant Acinetobacter baumannii producing carbapenemases in Korea. J Microbiol 2006;44(4):423-31.

14. Romao CM, Faria YN, Pereira LR, Asensi MD. Susceptibility of clinical isolates of multiresistant Pseudomonas aeruginosa to a hospital disinfectant and molecular typing. Mem Inst Oswaldo Cruz 2005; 100(5):541-8.

15. Chang MR, Carvalho NC, Oliveira AL, Moncada PM, Moraes BA, Asensi MD. Surveillance of pediatric infections in a teaching hospital in Mato Grosso do Sul, Brazil. Braz J Infect Dis. 2003; 7(2):149-60.
16. Lodise TP, Miller CD, Graves J et al. Clinical prediction tool to identify patients with Pseudomonas aeruginosa respiratory tract infections at greatest risk for multidrug resistance. Antimicrob Agents Chemother 2007; 51(2):417-22.

17. Zavascki AP, Barth AL, Fernandes JF, Moro AL, Goncalves AL, Goldani LZ. Reappraisal of Pseudomonas aeruginosa hospitalacquired pneumonia mortality in the era of metallo-beta-lactamase-mediated multidrug resistance: a prospective observational study. Crit Care 2006; 10(4):R114.

18. Sader HS, Gales AC, Pfaller MA et al. Pathogen frequency and resistance patterns in Brazilian hospitals: summary of results from three years of the SENTRY Antimicrobial Surveillance Program. Braz J Infect Dis 2001; 5(4):200-14.

19. Furtado GH, Martins ST, Machado AM, Wey SB, Medeiros EA. Clinical culture surveillance of carbapenem-resistant Pseudomonas aeruginosa and Acinetobacter species in a teaching hospital in Sao Paulo, Brazil: a 7-year study. Infect Control Hosp Epidemiol 2006; 27(11):1270-3.

20. Raja NS, Singh NN. Antimicrobial susceptibility pattern of clinical isolates of Pseudomonas aeruginosa in a tertiary care hospital. J Microbiol Immunol Infect 2007; 40(1):45-9.

21. Evans ME, Feola DJ, Rapp RP. Polymyxin B sulfate and colistin: old antibiotics for emerging multiresistant gram-negative bacteria. Ann Pharmacother 1999; 33(9):960-7.

22. Aubert D, Poirel L, Chevalier J, Leotard S, Pages JM, Nordmann P. Oxacillinase-mediated resistance to cefepime and susceptibility to ceftazidime in Pseudomonas aeruginosa. Antimicrob Agents Chemother 2001; 45(6):1615-20.

23. Giakkoupi P, Petrikkos G, Tzouvelekis LS, Tsonas S, Legakis NJ, Vatopoulos AC. Spread of integron-associated VIM-type metallo-beta-lactamase genes among imipenem-nonsusceptible Pseudomonas aeruginosa strains in Greek hospitals. J Clin Microbiol 2003; 41(2):822-5.

24. Graf T, Fuentefria DB, Corcao G. [Occurrence of multiresistant strains of Pseudomonas aeruginosa producing metallobeta-lactamase blaSPM-1 in clinical samples]. Rev Soc Bras Med Trop 2008; 41(3):306-8.

25. Carvalho AP, Albano RM, de Oliveira DN, Cidade DA, Teixeira LM, Marques Ede A. Characterization of an epidemic carbapenem-resistant Pseudomonas aeruginosa producing SPM-1 metallo-beta-lactamase in a hospital located in Rio de Janeiro, Brazil. Microb Drug Resist 2006; 12(2):103-8.

26. Goncalves DC, Lima AB, Leao LS, Filho JR, Pimenta FC, Vieira JD. [Detection of metallo-beta-lactamase in Pseudomonas aeruginosa isolated from hospitalized patients in Goiania, State of Goias]. Rev Soc Bras Med Trop. 2009; 42(4):411-4.

27. Figueiredo-Mendes CM, Sinto S, Mello-Sampaio JL et al. Pseudomonas aeruginosa clonal dissemination in Brazilian intensive care units. Enferm Infecc Microbiol Clin. 2005; 23(7):402-5. 